$15 \%$ and $28 \%$ of these patients had severe hepatotoxicity at 4 and 24 weeks, respectively. Serum levels of all enzymes increased significantly $(\mathrm{p}<0.05)$ with increased treatment duration. Univariate analysis revealed that the risk factor of developing severe hepatotoxicity was significantly $(\mathrm{p}<0.05)$ greater in patients $<30$ years, males, low BMI, low monthly income earners and patient on $\mathrm{AZT}+3 \mathrm{TC}+\mathrm{NVP}$ regimen. While multivariate analysis at $\mathrm{p}<0.09$ showed that age $<30$ years, Low BMI, low monthly income, or the use of AZT $+3 \mathrm{TC}+\mathrm{NVP}$ regimen were independent risk factors.

Conclusion Low BMI, $<30$ years, low monthly income and the use of $\mathrm{AZT}+3 \mathrm{TC}+\mathrm{NVP}$ regimen were identifiable risk factors for the development of severe hepatotoxicity. As such, these factors should be considered as important for strategy by clinicians to prevent hepatotoxicity.

\section{PO 8194 OPTIMISED INFORMED CONSENT FOR PARTICIPANTS IN A RANDOMISED CONTROLLED TRIAL IN RURAL UGANDA: A COMPARATIVE PROSPECTIVE COHORT STUDY}

\begin{abstract}
${ }^{1}$ James Ditai*, ${ }^{1}$ Jesca Kanyago, ${ }^{1}$ Rachael Nambozo, ${ }^{1}$ Nathan Kanyago, ${ }^{1} J u l i a n$ Abeso, ${ }^{2}$ Peter Olupot, ${ }^{3}$ Enitan Carrol, ${ }^{3}$ Melissa Gladstone, ${ }^{3}$ Andrew Weeks, ${ }^{4}$ Brian Faragher, ${ }^{3}$ Antonietta Medina-Lara, ${ }^{5} J u l i e$ Storr. 'Sanyu Africa Research Institute, Mbale, Uganda; ${ }^{2}$ Busitema University Faculty of Health Sciences, Mbale, Uganda; ${ }^{3}$ University of Liverpool, UK; ${ }^{4}$ Liverpool School of Tropical Medicine, UK; ${ }^{5}$ World Health Organization, Geneva, Switzerland
\end{abstract}

\subsection{6/bmjgh-2019-EDC.55}

Background Poor participant understanding of research information can be a problem in community interventional studies in rural African women where levels of illiteracy, dependency and compliance are high. We assessed the impact of alternative consent models on participants' understanding of the clinical trial information and its contribution to the informed consent process in rural Uganda.

Methods This was a prospective comparative cohort, nested within a pilot study of community distribution of alcoholbased hand rub to prevent neonatal sepsis (BabyGel). As part of the informed consent process, information about the trial was presented using one of three consent methods: standard researcher-read information; a 'slide show' using illustrated text on a flip chart; and a video showing the patient information sheet (PIS) being read as if by a newsreader in either English or the local language. In addition, all women received a written PIS in their preferred language. Each information method was used for 1 week of recruitment. Two days after recruitment, women's understanding of the clinical trial was evaluated.

Results A total of 30 pregnant women from 13 villages in Mbale participated in this study. Majority 90\% (27/30) were assessed for recall of trial information within planned 48 hours. The slide-show was the most popular, with a mean score not less than 4.2 highest [mean (sd) range: 4.8 (0.6) [45]] by women who had been recruited using any of the three models. The slide show was preferred by $63 \%$ of the women (19/30), compared with 17\% (5/30) and 20\% (6/30) who preferred the standard and video show message, respectively. Reasons given included the benefits of having pictures to aid understanding, and the logical progression of the information. Conclusion Our results suggest that a slide-show message is an effective and popular alternative way of presenting trial information to women in rural Uganda, many of whom had little or no literacy.

\section{PO 8208 CYTOKINE PROFILES IN SUDANESE CHILDREN PRESENTED WITH SEVERE MALARIA, UNCOMPLICATED MALARIA COMPARED TO HEALTHY COMMUNITY CONTROLS ACCORDING TO WHO CRITERIA}

${ }^{1}$ Hamdan Hamdan*, ${ }^{2}$ Muna El-Misbah, ${ }^{1}$ Tasneem El-Awad. ${ }^{1}$ Al-Neelain University, Faculty of Medicine, Khartoum, Sudan; ${ }^{2}$ Sudan International University, Khartoum, Sudan

\subsection{6/bmjgh-2019-EDC.56}

Background Immune system response to Plasmodium falciparum (P. falciparum) malaria infection outlines the disease course and outcome. This is attributed to variable production of cytokines that either promote (pro-inflammatory) or curtail (anti-inflammatory) the inflammatory process. Elucidating underlying immunological disease interactions may direct development of effective treatment and provide better understanding of the disease process.

Methods A case control study was conducted in Mohamed Elamin Paediatrics Hospital (March- August 2016) in Omdurman in central Sudan, an area that is characterised by unstable malaria transmission. The study aims to investigate the role/ interaction of cytokine profiles of gamma interferon (IFN- $\gamma$ ) and Interleukin-10 (IL-10) in children infected with P. falciparum malaria. Enzyme-linked immunosorbent assay was used to measure the concentrations of cytokines, IFN- $\gamma$ and IL-10, in serum from Sudanese children. Thirty-five children with complicated $P$. falciparum malaria were enrolled to the study; well-matched 35 uncomplicated $P$. falciparum malaria and another 35 healthy children were controls. Informed written consent was obtained from the parents or guardian. Complete blood count, blood urea and random blood glucose were measured by using standard laboratory procedures.

Results The concentrations of IFN- $\gamma$ and IL-10 levels were significantly higher in children with severe malaria compared to uncomplicated malaria and healthy control. There was a strong positive correlation observed between IL-10 and IFN- $\gamma$ $(\mathrm{r}=0.688 \mathrm{p}=<0.001$, as well as a strong positive correlation detected between IFN- $\gamma$ and urea levels $(r=0.73 ; \mathrm{p}=0.010)$. There was moderate correlation between IL-10 and urea $(\mathrm{r}=0.386 ; \mathrm{p}=<0.001)$. While negative moderate correlation was observed between IL-10 and haemoglobin levels $(\mathrm{r}=-0.316 ; \mathrm{p}=0.003)$, no correlation was detected between IFN- $\gamma$ and haemoglobin levels. All patients were discharged home in good condition.

Conclusion These results indicate both IFN- $\gamma$ and IL-10 are involved in shaping the course and outcome of the severe malaria in children.

\section{PO 8239 BASELINE ASSESSMENT OF LYMPHATIC FILARIASIS IN 18 COMMUNITIES IN WESTERN GHANA BEFORE THE IMPLEMENTATION OF TWICE-YEARLY TREATMENT}

Dziedzom De Souza*, Collins Stephen Ahorlu, Joseph Otchere, Sedzro Mensah, Sudan AduAmankwah, Daniel Boakye. Noguchi Memorial Institute for Medical Research, University of Ghana, Accra, Ghana

\subsection{6/bmjgh-2019-EDC.57}

Background Lymphatic filariasis (LF) is a neglected tropical disease targeted for elimination as a public health problem by 
2020, with the main strategy being treatment of entire endemic communities. Since the inception of the Global Programme for the Elimination of LF in 2000, tremendous progress has been made in many endemic countries. However, current observations point to the need for improved treatment regimen, frequency of treatment or drug delivery strategies in order to achieve the elimination goals in certain endemic areas. In this randomised trial, we evaluate the use of twiceyearly treatment with ivermectin and albendazole in 18 LFendemic communities in Ghana, where despite 15 years of yearly treatment the disease is still above the elimination thresholds.

Methods Following demographic data collection, Wuchereria bancrofti antigen, microfilaria and antibody prevalence were assessed in study participants using the Alere FTS kit, nucleopore filtration and Wb123 ELISA, respectively. The study assessed the perspectives of the communities'on persistent transmission of LF in view of implementing effective treatment uptake strategies.

Results The baseline assessments revealed antigen prevalence of $8.2 \%(95 \% \mathrm{CI}=6.8-9.8)$, with overall microfilaria prevalence of $1.2 \%$. Infections were higher in males and in individuals who spend significant amount of time outdoors for commercial activities. Barriers related to medication, personal, health system, disease and social structure were observed to affect mass drug administration compliance. Community members perceived that they were not susceptible to infection and this together with drug adverse effects strongly affect the ingestion of the drugs.

Conclusion While this trial is still in an early phase, the baseline assessments reveal programmatic challenges to the implementation of a twice-yearly treatment strategy for the control of LF which must be addressed to enhance implementation success.

\section{PO 8248 DETERMINANTS OF ACCEPTABILITY OF MALARIA RAPID DIAGNOSTIC TEST AMONG HEALTH WORKERS IN KINTAMPO NORTH MUNICIPALITY, GHANA}

${ }^{1}$ Michael Anaba*, ${ }^{1}$ Kenneth Ae-Ngebisi, ${ }^{2}$ Seth Owusu Agyei, ${ }^{3}$ Latifat Ibisomi. ${ }^{1}$ Kintampo Health Research Center, Kintampo, Ghana; ${ }^{2}$ University of Health and Allied Sciences, Hohoe, Ghana; ${ }^{3}$ University of the Witwatersrand, Johannesburg, South Africa

\subsection{6/bmjgh-2019-EDC.58}

Background Ghana rolled out the policy in 2013 with the use of malaria rapid diagnostic test (mRDT) promoted to facilitate diagnosis. However, health workers who are at the centre of mRDT implementation still treat half of febrile patients with negative mRDT results with antimalarial drugs, suggesting limited or lack of acceptability of the innovation.

Methods We conducted a cross-sectional study to examine determinants of mRDT among health workers in Kintampo North Municipality (KNM) in Ghana. Data were collected from 110 health workers in KNM involved in malaria management from February to April 2017. The survey tool was based on two frameworks - the Technology Acceptance Model (TAM) and Normalisation Process Theory (NPT). Acceptability was measured by ease of use, perceived usefulness and intention to use.

We hypothesised that acceptability was influenced by coherence, cognitive participation, collective action, reflexive monitoring and respondent characteristics. A composite acceptability score was computed from a 21-item questionnaire for each respondent. The respondents were divided into three groups of low, moderate and high acceptability for ordered logistic regression to examine the relationship between acceptability and its determinants.

Results The median acceptability score was 84(Q1, Q3:68, 103). About $34 \%$ of health workers had low acceptability while $37 \%$ and $29 \%$ had moderate and high acceptability respectively. In the multivariable analysis, coherence $(\mathrm{OR}=1.23, \quad 95 \% \mathrm{CI}=1.11-1.37), \quad$ cognitive participation $(\mathrm{OR}=1.35,95 \% \mathrm{CI}=1.10-1.66)$, health workers in rural health facilities $(\mathrm{OR}=6.99,95 \% \mathrm{CI}=1.82-26.84)$ and health workers with more than three years' experience $(\mathrm{OR}=5.53,95 \%$ $\mathrm{CI}=1.98-15.42$ ) were more likely to have high mRDT acceptability.

Conclusion Acceptability of mRDT was moderate among the majority. This can be improved by enhancing health workers' coherence on the benefits of mRDT through policy building or dissemination of information, promoting health workers' cognitive participation in the mRDT implementation process through recruitment of local 'champions' to promote 'buy-in' and providing incentives to health workers to embed and sustain the use of the health technology.

\section{PO 8249 SUBMICROSCOPIC PLASMODIUM FALCIPARUM INFECTIONS IN MATCHED PERIPHERAL, PLACENTAL AND UMBILICAL CORD BLOOD SAMPLES FROM CONGOLESE WOMEN AT DELIVERY}

${ }^{1}$ Yvon Mbouamboua*, ${ }^{1}$ Félix Koukouikila-Koussounda, ${ }^{2} J a c q u e s$ Van Helden, ${ }^{1}$ Francine Ntoumi. ${ }^{1}$ Fondation Congolaise pour la Recherche Médicale, Brazzaville, Republic of the Congo; ${ }^{2}$ Aix-Marseille University, Institute de Neurosciences des Systèmes, Marseille, France

\subsection{6/bmjgh-2019-EDC.59}

Background This cross-sectional study was conducted to characterise $P$. falciparum infections matched in peripheral, placental and cord blood among Congolese women at delivery receiving 1, 2 or more doses of sulfadoxine-pyrimethamine. The cross-sectional study was conducted in a Southern district of Brazzaville, Republic of the Congo, between March 2014 and April 2015.

Methods Peripheral and placental blood samples were collected for P. falciparum infection investigation by microscopy and nested polymerase chain reaction (PCR), using P. falciparum merozoite surface protein-2 (msp2) gene as marker.

Results Of the 370 pregnant women recruited, only $7.3 \%$ peripheral and $2.7 \%$ placental blood samples were found smear-positive for $P$. falciparum by microscopy. All isolates from cord blood were microscopy-negative. However, the prevalences of submicroscopic $P$. falciparum infections (detectable only by PCR) were $25.4 \%, 16.7 \%$ and $9.4 \%$ in peripheral, placental and cord blood respectively. The frequency of 3D7 msp2 alleles was the highest $(>60 \%)$ whatever the blood considered. We found a high prevalence of submicroscopic infection in pregnant women associated with a high genetic diversity of $P$. falciparum isolates. The multiplicity of infection ranged between 1.2 and 1.4 irrespective of the blood compartment, and it showed no significant association with maternal age $(p=0.3)$, gravidity $(p=0.1)$ or sulfadoxinepyrimethamine $(p=0.3)$.

Conclusion In summary, this study showed that there is a high prevalence of submicroscopic infection and a high genetic diversity of Plasmodium falciparum strains in Congo. This 